\title{
Teste de Permeabilidade de Vias Aéreas Pré-Extubação: Comparação entre Três Métodos em Ventilação Espontânea*
}

\author{
Cuff Leak Test Preextubation: Comparison between \\ Three Methods in Spontaneous Ventilation
}

\author{
Samantha da Silva Souza', Luciana Castilho de Figueiredo², \\ Cristina Aparecida Veloso Guedes ${ }^{2}$, Sebastião Araújo ${ }^{3}$
}

\section{RESUMO}

JUSTIFICATIVA E OBJETIVOS: O teste de permeabilidade avalia obstrução de via aérea superior e é classicamente realizado em modo assistido-controlado de ventilação mecânica. O objetivo deste estudo foi analisar este teste em ventilação espontânea, através de três diferentes métodos e compará-los.

MÉTODO: Vinte pacientes intubados foram submetidos a três diferentes formas do teste de permeabilidade, todos em ventilação espontânea: com o ventilômetro e o paciente conectado ao ventilador (teste 1); através do display do ventilador mecânico (teste 2); e com o ventilômetro e o paciente desconectado do ventilador (teste 3). O vazamento ao redor do tubo traqueal (TT) foi definido como a porcentagem decorrente da diferença entre o volume-corrente inspirado (balonete insuflado) e expirado (balonete desinsuflado). Foram avaliadas as diferenças entre os três testes, bem como correlacionado a porcentagem de vazamento entre os testes com três variáveis: pressão do balonete, diâme-

1. Fisioterapeuta; Aluna do Curso de Aprimoramento em Fisioterapia Respiratória em Unidade de Terapia Intensiva da UNICAMP

2. Fisioterapeuta Supervisora da Unidade de Terapia Intensiva da UNICAMP

3. Professor Assistente Doutor do Departamento de Cirurgia da FCM-UNICAMP e Assistente da Unidade de Terapia Intensiva da UNICAMP

* Recebido da Unidade de Terapia Intensiva do Hospital de Clínicas da Universidade Estadual de Campinas (HC- UNICAMP), Campinas, SP

Apresentado em 19 de abril de 2007

Aceito para publicação em 31 de julho de 2007

Endereço para correspondência:

Samantha da Silva Souza

Av. Monteiro Lobato, 267/12 - Vila Valença

11380-500 São Vicente, SP

E-mail: samanthasz@hotmail.com

(C)Associação de Medicina Intensiva Brasileira, 2007 tro do TT e tempo de intubação.

RESULTADOS: Houve diferença significativa $(p<0,05)$ de vazamento entre os testes 1 e 2 em relação ao teste 3 no geral e relacionado à intubação, com período inferior a 48h e pressão de balonete abaixo de $20 \mathrm{cmH}_{2} \mathrm{O}$. Em relação ao diâmetro do tubo, houve diferença apenas entre os testes 2 e 3 para tubos de $8,5 \mathrm{~mm}$.

CONCLUSÕES: O teste de permeabilidade em ventilação espontânea parece ser mais fidedigno quando realizado com o paciente conectado ao ventilador mecânico, mas novos estudos devem ser realizados para a determinação da real contribuição do teste em ventilação espontânea para a predição de edema de laringe.

Unitermos: Edema laríngeo, intubação traqueal, ventilação artificial, testes de função respiratória, teste de vazamento do balonete.

\section{SUMMARY}

BACKGROUND AND OBJECTIVES: The cuff leak test aims to evaluate the presence of airway obstruction and normally is carried through in the controlled mode of mechanical ventilation. The objective of this study was to evaluate the cuff leak in patients breathing spontaneously, across three different methods, and to compare them.

METHODS: Twenty intubated patients had been submitted to three different forms of cuff leak test, all of them in spontaneous respiration: measuring air leak buy using a ventilometer and with the patient connected to the mechanical ventilator (test 1); through the display of the mechanical ventilator (test 2); and with ventilometer and the patient detached from the mechanical ventilator (test 3). The air leak around the tracheal tube (TT) was defined as the percentage difference between the inspired tidal volume (insufflated cuff) and exhaled (deflated cuff). The air leak differences betwe- 
en the three tests were evaluated, as well as their correlations to three variables: cuff pressure, $\Pi$ diameter and intubation time.

RESULTS: Statistically significant $(p<0.05)$ air leak difference was observed between the tests 1 and 2 in relation to the test 3 in the general and regarding time intubation below $48 \mathrm{~h}$ and cuff pressure below 20 $\mathrm{cmH}_{2} \mathrm{O}$. Regarding the tube diameter, it had been difference only between tests 2 and 3 for $8.5 \mathrm{~mm}$ tubes.

CONCLUSIONS: The cuff leak test in spontaneous ventilation seems to be more accurate when the patient is connected to the mechanical ventilator, and that additional studies are needed to determine the real contribution of the test in this ventilation mode to predict laryngeal edema.

Key Words: artificial ventilation, cuff leak test, tracheal intubation, laryngeal edema, ventilatory function tests.

\section{INTRODUÇÃO}

A intubação traqueal permite a adaptação do suporte ventilatório para pacientes com incapacidade de manutenção adequada da ventilação, constituindo um tratamento padrão de suporte de vida ${ }^{1-3}$.

O profissional deve ser capaz de decidir se o paciente pode tolerar a remoção do suporte ventilatório, para então remover o tubo traqueal $(\mathrm{TT})^{4}$. Dificuldade na extubação é um evento raro, mas pode ser uma complicação perigosa e ocasionalmente fatal no processo de ventilação mecânica. A taxa de mortalidade resultante de falha na extubação é de até $50 \%$, o que indica o potencial de gravidade desse tipo de evento ${ }^{5-6}$.

As complicações relacionadas à intubação, decorrentes de lesões à laringe, faringe e traquéia podem ocorrer tanto durante a intubação quanto após a extubação ${ }^{7}$. O desenvolvimento de formas práticas e preditivas que possam otimizar a decisão de extubar um paciente exige conhecimento dos fatores de risco e causas de suas falhas ${ }^{4}$. Lesões de laringe determinam a ocorrência de obstrução das vias aéreas ${ }^{8}$ e a intubação traqueal causa complicações, particularmente, nessa estrutura anatômica.

$\mathrm{O}$ edema de laringe, que normalmente ocorre logo após a extubação ${ }^{5}$, é complicação que tem sido associada ao trauma durante a intubação, uso de tubos com diâmetros maiores, hiperinsuflação do balonete, intubação oral e alterações do tubo traqueal ${ }^{9-10}$.

A necessidade de re-intubação após falha na extubação está associada com morbidade e mortalidade sig- nificativas ${ }^{11}$, além de aumento do tempo de permanência na UTI ${ }^{4,12}$ e da taxa de traqueostomia ${ }^{12-14}$. Assim, a identificação precoce de pacientes com necessidade de re-intubação, a conseqüente re-instituição do suporte ventilatório é fundamental ${ }^{4,11,15}$.

O teste de permeabilidade de vias aéreas foi proposto como ferramenta simples para a detecção de edema de laringe ${ }^{16-18}$, mostrando ser bom indicador ${ }^{3}$. Este teste foi desenvolvido por Potgieter e Hammond ${ }^{19}$, do Hospital Groot Schwer, na África do Sul, e consistia basicamente em desinsuflar o balonete e ocluir digitalmente o tubo traqueal a fim de avaliar a presença de vazamento de ar ao seu redor, o que permitia a determinação indireta de desobstrução da via aérea superior antes da extubação ${ }^{18,20,21}$.

Entretanto, o teste foi ganhando forma mais apurada e, em 1996, Miller e Cole ${ }^{12}$ apresentaram um protocolo útil e de fácil reprodutibilidade, passando assim a ser utilizado em outros estudos. Os autores propuseram que, imediatamente antes do teste, adapte-se o paciente ao modo assistido-controlado na ventilação mecânica. Após isso, aspiram-se secreções traqueais e orais. A aspiração reduz a quantidade de secreções aderidas ao tubo, que podem influenciar no volume de vazamento ${ }^{16}$. Ainda com o balonete insuflado, registram-se o volume-corrente $\left(\mathrm{V}_{T}\right)$ inspirado e expirado, além da medida da pressão do balonete através de um manômetro. Em seguida, desinsufla-se o balonete e mede-se $\circ V_{T}$ expirado em três ciclos ventilatórios subseqüentes, obtendo-se então uma média dos três menores valores. Por fim, obtém-se o volume de vazamento, definido como a diferença entre $\circ V_{T}$ inspirado e a média dos $\mathrm{V}_{\mathrm{T}}$ expirados ${ }^{22}$.

Embora seja um protocolo bem definido, submeter pacientes ao modo assistido-controlado na ventilação mecânica pode necessitar de sedação e até mesmo bloqueio neuromuscular ${ }^{23}$, processo difícil de ser realizado em pacientes prestes a serem extubados. Entretanto, não há disponível na literatura estudos sobre a realização do teste de permeabilidade em pacientes sob outras modalidades de ventilação mecânica.

O objetivo deste estudo foi verificar a porcentagem de vazamento do teste de permeabilidade, através de três diferentes métodos, com o paciente ventilando espontaneamente e não em ventilação controlada como já proposto por Miller e Cole e compará-los entre si, a fim de se identificar suas diferenças e avaliar se todas as formas do teste podem ser utilizadas com o mesmo objetivo. 


\section{MÉTODO}

Foi realizado estudo prospectivo incluindo 20 pacientes adultos, com idade $\geq 18$ anos, intubados e ventilados mecanicamente, internados na UTI do Hospital das Clínicas da Universidade Estadual de Campinas (UNICAMP) e que estivessem aptos a serem extubados. Foram excluídos do estudo os pacientes com idade inferior a 18 anos, traqueostomizados e/ou portadores de anormalidades traqueolaríngeas, tais como neoplasia, estenose, paralisia de cordas vocais e traqueomalácia. Também foram excluídos do estudo os pacientes cujas extubações foram concluídas sem a realização prévia do teste de permeabilidade de vias aéreas.

O protocolo do presente estudo foi aprovado pelo Comitê de Ética e Pesquisa da Faculdade de Ciências Médicas da UNICAMP, sob o parecer $n^{\circ} 301 / 2006$. Foi obtido um termo de consentimento livre e esclarecido dos pacientes ou seus familiares.

Foi construída uma ficha de coleta de dados para facilitar a análise estatística.

A pressão arterial média (PAM), a freqüência cardíaca $(F C)$, a freqüência respiratória $(F R)$ e a oximetria de pulso foram monitorizadas antes e durante a realização do teste, a fim de ser interrompida, em caso de instabilidade hemodinâmica e/ou respiratória do paciente. Nas 24 horas subseqüentes à extubação, foi observada ocorrência de edema de laringe, caracterizado pela presença de estridor inspiratório associado a elevação de freqüência respiratória e esforço respiratório.

Os ventiladores usados para o estudo foram o Raphael ${ }^{\circledR}$ (Hamilton Medical) e o Evita $2^{\circledR}$ (Dräger). As pressões de balonete do TT foram medidas com um manômetro específico (Portex CE 0473). Para a medida do volume-minuto $\left(\mathrm{V}_{\mathrm{E}}\right)$ obtido com os testes de permeabilidade de vias aéreas, foi utilizado um ventilômetro digital (AINCA 295).

\section{Testes Permeabilidade de Vias Aéreas}

O vazamento ao redor do TT foi avaliado em cada paciente imediatamente antes da extubação eletiva. Nesse momento, os pacientes estavam com parâmetros considerados mínimos para extubação e foram mantidos nesta forma de ventilação durante os testes. Previamente, foi realizada higiene brônquica e de vias aéreas superiores, bem como medida a pressão do balonete.

A porcentagem de vazamento foi mensurada utilizan- do-se três diferentes métodos:

- Teste 1: com o paciente conectado ao ventilador mecânico, conectava-se o ventilômetro ao ramo inspiratório do ventilador e media-se $V_{E}$ e FR. Em seguida, conectava-se o ventilômetro ao ramo expiratório do ventilador; realizava-se desinsuflação do balonete e media-se $V_{E}$ e FR. $O$ balonete era então reinsuflado aos valores prévios;

- Teste 2: realizado apenas nos pacientes ventilados com o Raphael ${ }^{\circledR}$, ventilador que dispõe do recurso de calcular a porcentagem de vazamento e mostrá-la em seu display. A porcentagem de vazamento determinada pelo ventilador era registrada após desinsuflação do balonete;

- Teste 3: desconectava-se o paciente da ventilação mecânica e acoplava-se o ventilômetro ao TT a fim de se verificar $V_{E}$ e FR inspiratórios. Em seguida, o balonete era desinsuflado e o ventilômetro conectado ao TT a fim de verificar $\mathrm{V}_{\mathrm{E}}$ e FR expiratórios. Após tais medidas, o balonete era reinsuflado e o paciente reconectado ao ventilador mecânico.

Cada teste foi realizado apenas uma vez. Os testes foram realizados subseqüentemente e em intervalos de um minuto, em todos os pacientes.

Obtidas todas as medidas, realizava-se o cálculo de $\mathrm{V}_{\mathrm{T}}\left(\mathrm{V}_{\mathrm{E}} / \mathrm{FR}\right)$ inspiratório e expiratório. $\mathrm{O}$ vazamento ao redor do TT foi definido como a porcentagem decorrente da diferença entre o $V_{T}$ inspiratório com o balonete insuflado e o $V_{T}$ expiratório com o balonete desinsuflado.

\section{Análise Estatística}

Para avaliar os dados foram utilizados testes de análise de variância. As amostras que apresentaram distribuição normal e variâncias iguais foram submetidas ao ANOVA, sendo que o teste aplicado para a verificação de normalidade foi o teste Kolmogorov-Smirnov (Lilliefors). Quando houve diferença significativa na ANOVA, foi aplicado o teste de Tukey a fim de localizar entre quais grupos estavam presentes as diferenças. Nos casos em que as amostras apresentaram falhas na normalidade, variâncias desiguais e/ou grupos muito pequenos, optou-se por um procedimento não paramétrico, o teste de Kruskal-Wallis, que foi seguido pelo método de Dunn para comparação entre os grupos quando constatada diferença significativa na análise.

O nível significativo adotado foi de $5 \%$, ou seja, $p<$ 0,05 .

O fluxograma do estudo está representado na figura 1. 
TESTE DE PERMEABILIDADE DE VIAS AÉREAS PRÉ-EXTUBAÇÃO:

COMPARAÇÃO ENTRE TRÊS MÉTODOS EM VENTILAÇÃO ESPONTÂNEA

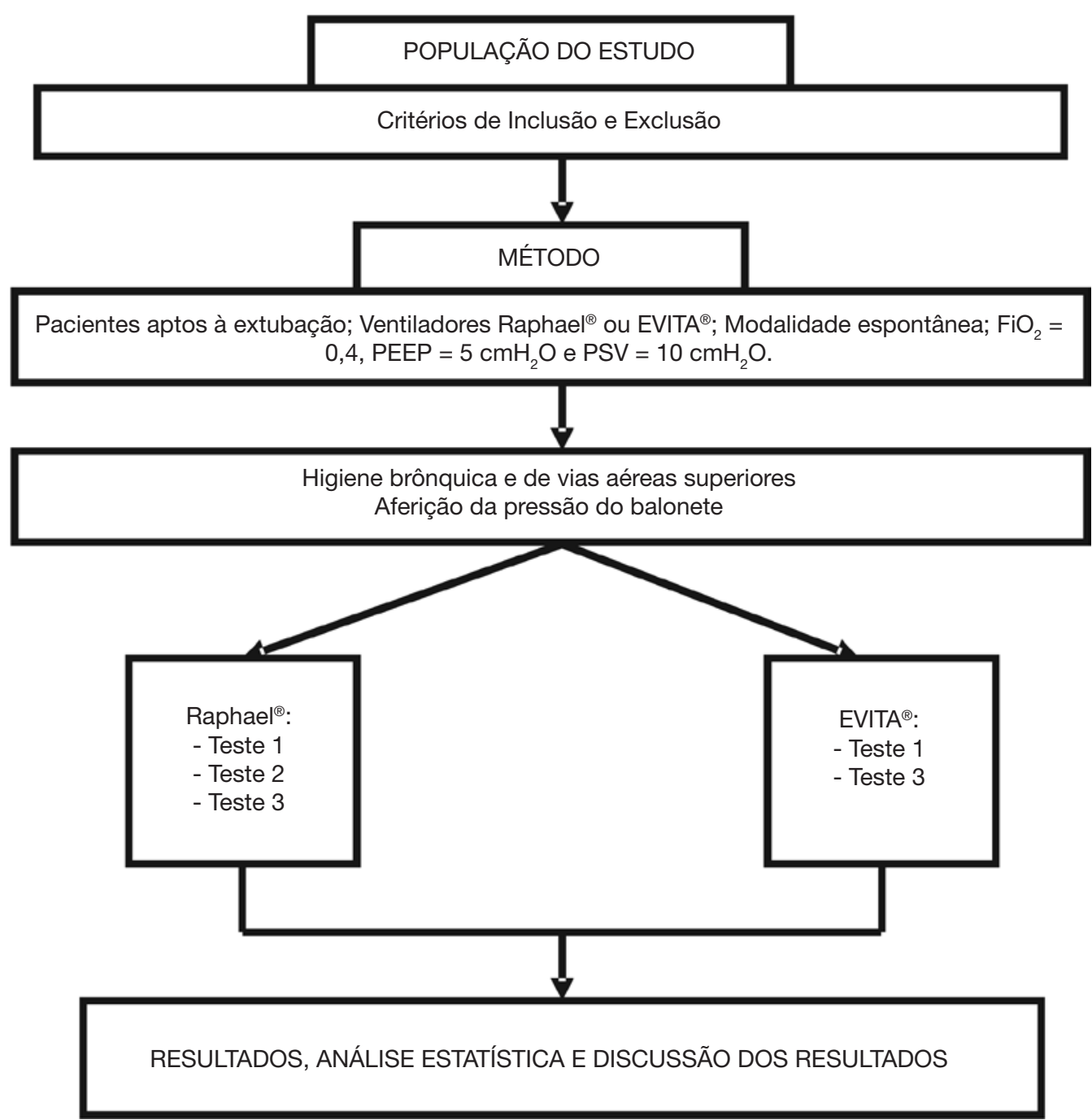

Figura 1 - Fluxograma do Estudo.

$\mathrm{FiO}_{2}=$ fração inspirada de oxigênio; $\mathrm{PEEP}=$ pressão positiva expiratória final; $\mathrm{PSV}$ = ventilação com pressão de suporte; $\mathrm{cmH}_{2} \mathrm{O}=$ centímetros de água.

\section{RESULTADOS}

As características demográficas da população estudada estão apresentadas na tabela 1, enquanto o comportamento geral do vazamento apresentado pelos três tipos de testes está apresentado na figura 2. Esta figura sugere maior variação do teste 3 , fato confirmado na figura 3 , que mostra os valores de p para a comparação entre os três testes.
Tabela 1 - Características Demográficas da População Estudada $(n=20)$.

\begin{tabular}{lc}
\hline Características & Valores \\
\hline Sexo & Masculino $(n=11)$ \\
& Feminino $(n=9)$ \\
Idade (anos) & $48,8 \pm 15,25$ \\
Diâmetro do tubo traqueal & $7,5(n=3)$ \\
$(\mathrm{mm})$ & $8(\mathrm{n}=5)$ \\
& $8,5(\mathrm{n}=9)$ \\
Tempo de intubação & $9(\mathrm{n}=3)$ \\
(horas) & $<48(\mathrm{n}=16)$ \\
Pressão do balonete & $\geq 48(\mathrm{n}=4)$ \\
(cmH $\left.{ }_{2} \mathrm{O}\right)$ & até $20(\mathrm{n}=10)$ \\
& $21-40(\mathrm{n}=6)$ \\
Diagnóstico de admissão & $\geq 41(\mathrm{n}=4)$ \\
na UTI & PO de cirurgia cardíaca $(\mathrm{n}=10)$ \\
& PO de cirurgia abdominal $(\mathrm{n}=4)$ \\
& Outras causas $(\mathrm{n}=3)$ \\
\hline
\end{tabular}

$\mathrm{mm}=$ milímetros $; \mathrm{cmH}_{2} \mathrm{O}=$ centímetros de água; $\mathrm{PO}$ = pós-operatório. 


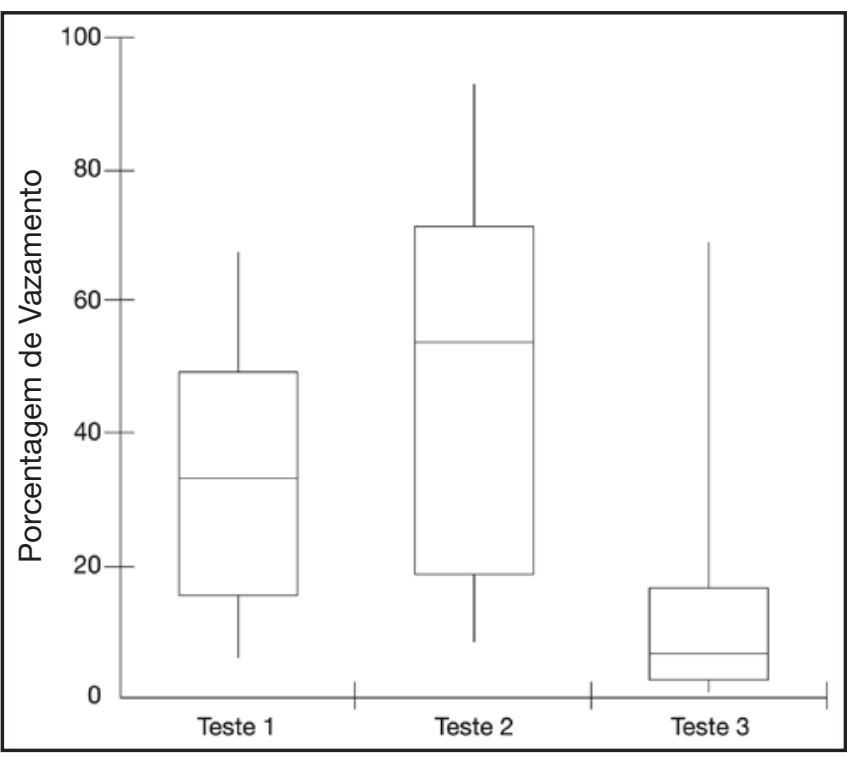

Figura 2 - Comportamento Geral da Porcentagem de Vazamento nos Três Testes de Permeabilidade.

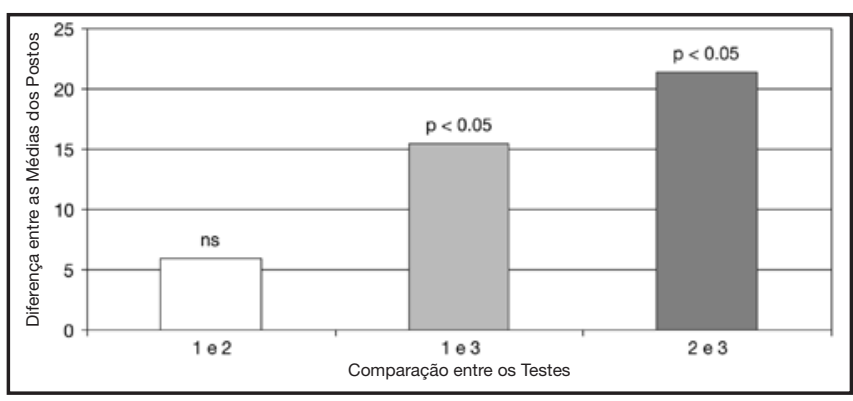

Figura 3 - Diferença de Vazamento entre os Três Testes de Permeabilidade Kruskal-Wallis e método de Dunn.

É importante ressaltar também que os volumes-correntes inspiratórios e expiratórios encontrados no teste 1 foram superiores aos encontrados no teste 3 (exclui-se desta comparação o teste 2, já que a porcentagem de vazamento foi dada pelo ventilador). A análise estatís tica mostrou diferença na média do volume-corrente inspiratório entre os testes 1 e 3 $(677,2 \pm 126,6 \mathrm{~mL}$ e $449,8 \pm 180,5 \mathrm{~mL}$, respectivamente; $p=0,0001$ ), mas não evidenciaram diferença estatística na média de volume-corrente expiratório entre os dois testes $(450,1 \pm 164,8 \mathrm{~mL}$ e $392,6 \pm$ $145,7 \mathrm{~mL} ; \mathrm{p}=0,25$ ).

A comparação entre a porcentagem de vazamento nos três tipos de testes e a duração da intubação mostrou diferença estatisticamente significativa apenas para pacientes ventilados mecanicamente por período inferior à 48h (Figura 4).

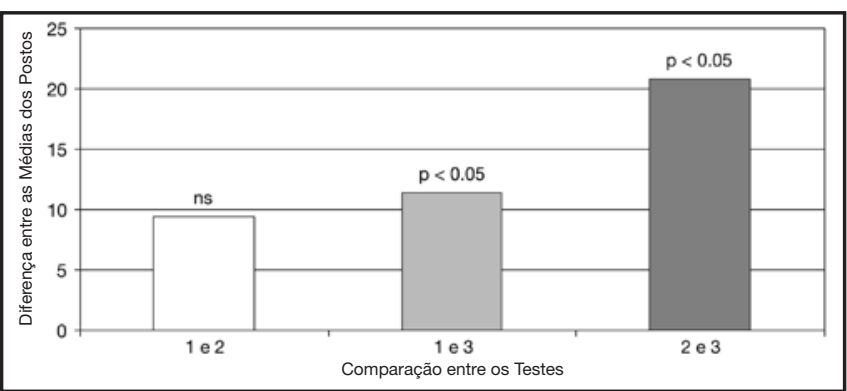

Figura 4 - Diferença de Vazamento entre os Três Testes de Permeabilidade em Relação às Intubações com menos de 48 Horas de Duração

Kruskal-Wallis e método de Dunn; testes 1 e 3: $n=16$; teste 2: $n=10$.

A análise inferencial intra e entre grupos, realizada contrapondo-se o diâmetro de tubo traqueal e a porcentagem de vazamento, evidenciou diferença estatisticamente significativa apenas na comparação entre os testes 2 e 3 em tubos de diâmetro 8,5 mm (Figura 5).

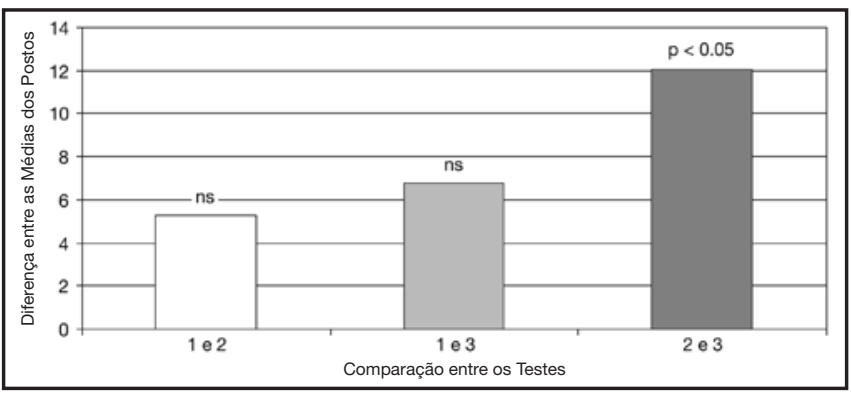

Figura 5 - Comparação entre a Porcentagem de Vazamento dos Diferentes Testes em Relação ao Diâmetro 8,5 mm de Tubo Traqueal

Kruskal-Wallis e método de Dunn; testes 1 e $3: n=9$; teste $2: n=8$.

A comparação entre os três tipos de testes e a pressão do balonete evidenciou diferenças estatisticamente significativas apenas para comparação entre os testes 1 e 3 e 2 e 3, somente para pressões abaixo de 20 $\mathrm{cmH}_{2} \mathrm{O}$ (Figura 6).

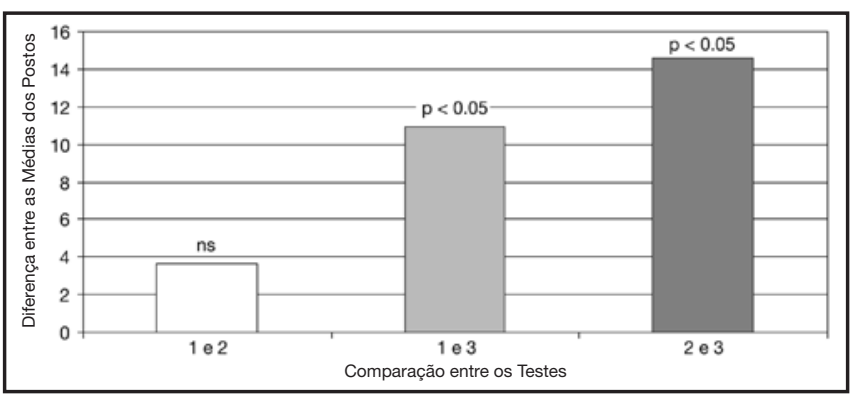

Figura 6. Comparação entre as Porcentagens de Vazamento Obtidas com Pressões de Balonete abaixo de $20 \mathrm{cmH}_{2} \mathrm{O}$, nos três Testes

Kruskal-Wallis e método de Dunn; testes 1 e $3: n=10$; teste 2: $n=9$. 
A parcela da amostra que apresentou valor de vazamento abaixo de $12 \%$, considerado limiar para a ocorrência de edema de laringe, está representada na tabela 2. A menor média de vazamento foi encontrada no teste 3.

Tabela 2 - Comparação entre a Porcentagem de Vazamento da População que Apresentou Taxa de Vazamento Inferior a 12\% em cada Teste

\begin{tabular}{ccc}
\hline Testes & $\mathrm{n}$ & Média de Vazamento $\pm \mathrm{DP}$ \\
\hline 1 & 4 & $7,27 \% \pm 2,3 \%$ \\
2 & 2 & $9 \% \pm 1 \%$ \\
3 & 12 & $3,97 \% \pm 2,19 \%$ \\
\hline
\end{tabular}

$\mathrm{DP}=$ desvio padrão

Dentre os pacientes que apresentaram porcentagem de vazamento acima de $12 \%$, o teste 3 também foi o que obteve menores valores, com média \pm desviopadrão igual a $29,31 \% \pm 18.95 \%$, contrastando com $40,8 \% \pm 16,5 \%$ no teste 1 e $53,5 \% \pm 27,6 \%$ no teste 2 . A análise estatística evidenciou diferença significativa $(p<0,05)$ para a comparação entre os testes 2 e 3.

Apenas um paciente apresentou menos de $12 \%$ de vazamento nos três tipos de testes de permeabilidade de vias aéreas.

Não houve diferença significativa entre as porcentagens de vazamento dos diferentes grupos de pressão do balonete, tempo de intubação ou pressão do balonete. Nenhum dos pacientes necessitou de re-intubação por edema de laringe.

\section{DISCUSSÃO}

Por diminuir o diâmetro do trato respiratório, o edema de laringe aumenta a resistência das vias aéreas e, conseqüentemente, o trabalho ventilatório ${ }^{4,9,16}$. Embora seja considerada complicação relativamente incomum $^{19,24}$, a incidência de estridor pós-extubação parece variar entre $2 \%$ e $36,8 \%$ 7,10,18,20,21,24, dependendo do estado dos pacientes e da população estudada ${ }^{25}$. De modo geral, a probabilidade de qualquer seqüela traqueolaríngea aumenta com o tempo de intubação ${ }^{1}$, embora outros fatores estejam associados ${ }^{26}$. Na população estudada não houve ocorrência de edema de laringe, talvez porque a maior parte dos pacientes $(80 \%)$ estivesse intubado por período inferior à $48 \mathrm{~h}$. Além disso, este teste parece não ser preditivo de edema de laringe em pacientes intubados por curto espaço de tempo $(<48 \mathrm{~h})^{27}$. Como nenhum dos pacientes do estudo não desenvolveu edema de laringe, não foi possível confirmar tal suspeita.
Altas pressões no balonete também estão relacionadas com maior probabilidade de desenvolvimento de estridor pós-extubação ${ }^{20}$. Nos resultados do presente estudo, mesmo os pacientes com altas pressões de balonete não desenvolveram edema de laringe. Também não houve relação significativa entre a pressão do balonete e a porcentagem de vazamento. A mesma associação ocorreu com o diâmetro do tubo traqueal, onde a maior porcentagem de vazamento ocorreu com tubos de diâmetros maiores $(8,5 \mathrm{~mm})$.

Outro fator a ser considerado é a divergência entre os valores dos testes 1 e 2 em relação ao teste 3 , o que pode ser decorrente do fato de o paciente estar sem o auxílio da pressão positiva durante este último teste. Embora $40 \%$ da amostra tenham apresentado vazamento acima de $12 \%$ no teste 3 , tais valores continuaram inferiores em relação aos demais testes; e nenhum dos pacientes evoluiu com edema de laringe e necessidade de re-intubação. Estes resultados sugerem que os valores de vazamento necessários para predizer edema de laringe, em testes de permeabilidade de vias aéreas, realizados com o paciente desconectado do ventilador possam ser muito menores do que aqueles determinados para o teste realizado sob ventilação mecânica, estipulando em $12 \%$ de vazamento ${ }^{21}$.

De modo geral, acredita-se que, quando há vazamento ao redor do tubo, após desinsuflação do balonete, é possível evoluir a extubação com sucesso ${ }^{28}$, pois as trocas gasosas serão possíveis ${ }^{23}$. Por outro lado, ausência de tosse (que comumente se segue à desinsuflação do balonete) ${ }^{25}$ ou baixo vazamento ao redor do tubo parecem ser bons preditivos de estridor ${ }^{18,20-22}$.

Entretanto, ausência de vazamento ao redor do TT, quando o balonete é desinsuflado, não significa que o tubo não possa ser removido com sucesso. Muitos pacientes com ausência de vazamento ao redor do TT são extubados com segurança, o que sugere falha na especificidade do teste ${ }^{28}$. Estas afirmações estão de acordo com o presente estudo, pois mesmo os pacientes que apresentaram baixa porcentagem de vazamento ao redor do tubo não evoluíram com edema de laringe.

De qualquer forma, a eficácia do teste é superior à ausculta da base do pescoço em busca da identificação de vazamento audível ${ }^{21}$.

Alguns fatores, todavia, podem limitar os achados do presente estudo. O primeiro destes fatores é o fato de que, mesmo tendo sido os testes realizados um imediatamente após o outro, houve variação de volume-minuto entre eles, já que os pacientes estavam ventilando 
espontaneamente. Segundo, a população investigada foi relativamente pequena, o que pode ter prejudicado a análise dos resultados. Além disso, é importante ressaltar a necessidade de estudos mais amplos, que incluam pacientes que tenham evoluído com edema de laringe, a fim de que se possa comparar a porcentagem de vazamento apresentado pelos pacientes ventilando espontaneamente, àquela apresentada por pacientes sob modalidade controlada de ventilação mecânica.

\section{CONCLUSÃO}

O teste de permeabilidade realizado em ventilação espontânea parece ser mais fidedigno quando realizado com o paciente conectado ao ventilador mecânico, visto que a porcentagem de vazamento obtida com o paciente despressurizado foi significativamente menor em relação às formas em que o paciente estava conectado ao ventilador.

Entretanto, acredita-se que sejam necessários novos estudos para comparar o vazamento obtido no modo clássico de mensuração de vazamento (sob ventilação controlada) ao vazamento obtido em ventilação espontânea, para que se possa avaliar a real contribuição do teste realizado em ventilação espontânea para a determinação de edema de laringe.

\section{ABREVIAÇÕES}

$\mathrm{cmH}_{2} \mathrm{O}$ : Centímetros de água

$\mathrm{FiO}_{2}$ : Fração inspirada de oxigênio

FR: Freqüência respiratória

PO: Pós-operatório

PEEP: Pressão positiva expiratória final

PSV: Ventilação com pressão de suporte

TT: Tubo traqueal

$\mathrm{V}_{\mathrm{E}}$ : Volume-minuto

$\mathrm{V}_{\mathrm{T}}$ : Volume-corrente

\section{REFERÊNCIAS}

01. Whited RE - A prospective study of laryndotracheal sequelae in longterm intubation. Laryngoscope, 1984;94:367-377.

02. Colice GL, Stukel TA, Dain B - Laryngeal complications of prolonged intubation. Chest, 1989;96:877-884.

03. Cheng $\mathrm{KC}$, Hou CC, Huang $\mathrm{HC}$ et al - Intravenous injection of methylprednisolone reduces the incidence of postextubation stridor in intensi- ve care unit patients. Crit Care Med, 2006;34:1345-1350.

04. Epstein SK - Decision to extubate. Intensive Care Med, 2002;28:535546.

05. Hartley M, Vaughan RS - Problems associated with tracheal extubation Br J Anaesth, 1993;71:561-568.

06. Demling RH, Read T, Lind LJ et al - Incidence and morbidity of extubation failure in surgical intensive care patients. Crit Care Med, 1988;16:573-577.

07. Ho LI, Harn HJ, Lien TC et al - Postextubation laryngeal edema in adults. Risk factor evaluation and prevention by hydrocortisone. Intensive Care Med, 1996;22:933-936.

08. Kastanos N, Estopa Miro R, Marin Perez A et al - Laryngotracheal injury due to endotracheal intubation: incidence, evolution, and predisposing factors. A prospective long-term study. Crit Care Med, 1983;11:362-367.

09. McCulloch TM, Bishop MJ - Complications of translaryngeal intubation. Clin Chest Med, 1991;12:507-521.

10. Darmon JY, Rauss A, Dreyfuss D et al - Evaluation of risk factors for laryngeal edema after tracheal extubation in adults and its prevention by dexamethasone. A placebo-controlled, double-blind, multicenter study. Anesthesiology, 1992;77:245-251.

11. Sandhu RS, Pasquale MD, Miller $\mathrm{K}$ et al - Measurement of endotracheal tube cuff leak to predict postextubation stridor and need for reintubation. J Am Coll Surg, 2000;190:682-687.

12. Miller RL, Cole RP - Association between reduced cuff leak volume and postextubation stridor. Chest, 1996;110:1035-1040.

13. Pettignano R, Holloway SE, Hyman D et al - Is the leak test reproducible? South Med J, 2000;93:683-685.

14. Engoren $\mathrm{M}$ - Evaluation of the cuff leak test in a cardiac surgery population. Chest, 1999;116:1029-1031.

15. Epstein SK., Ciubotaru RL - Independent effects of etiology of failure and time to reintubation on outcome for patients failing extubation. Am J Respir Crit Care Med, 1998;158:489-493.

16. Epstein SK, Ciubotaru RL, Wong JB - Effect of failed extubation on the outcome of mechanical ventilation. Chest, 1997;112:186-192.

17. Torres A, Gatell JM, Aznar E et al - Re-intubation increases the risk of nosocomial pneumonia in patients needing mechanical ventilation. Am J Respir Crit Care Med, 1995;152:137-141.

18. Dupont $\mathrm{H}$, Le Port $\mathrm{Y}$, Paugham-Burtz $\mathrm{C}$ et al - Reintubation after planned extubation in surgical ICU patients: a case-control study. Intensive Care Med, 2001;27:1875-1880.

19. Santos PM, Afrassiabi A, Weymuller EA Jr - Risk factors associated with prolonged intubation and laryngeal injury. Otolaryngol Head Neck Surg, 1994;111:453-459.

20. De Bast Y, De Backer D, Moraine JJ et al - The cuff leak test to predict failure of tracheal extubation for laryngeal edema. Intensive Care Med, 2002;28:1267-1272.

21. Prinianakis G, Alexopoulou C, Mamidakis E et al - Determinants of the cuff leak test: a physiological study. Crit Care, 2005;9:R24-R31.

22. Chung YH, Chao TY, Chiu CT et al - The cuff leak test is a simple tool to verify severe laryngeal edema in patients undergoing long-term mechanical ventilation. Crit Care Med, 2006;34:409-414.

23. Potgieter PD, Hammond JM - "Cuff" test for safe extubation following laryngeal edema. Crit Care Med, 1988;16:813-818.

24. Jaber S, Chanques G, Matecki S et al - Post-extubation stridor in intensive care unit patients. Risk factors evaluation and importance of the cuff leak test. Intensive Care Med, 2003;29:69-74.

25. Rothaar RC, Epstein SK - Extubation failure: magnitude of the problem, impact on outcomes, and prevention. Curr Opin Crit Care, 2003;9:59-66.

26. Marik PE - The cuff leak test as a predictor of postextubation stridor: a prospective study. Respir Care, 1996;41:509-511.

27. Maury E, Guglielminotti J, Alzieu M et al - How to identify patients with no risk for postextubation stridor? J Crit Care, 2004;19:23-28.

28. Fisher MM, Raper RF - The 'cuff leak' test for extubation. Anaesthesia, 1992:47:10-12. 